\title{
Perfiles clínicos del paludismo no complicado por Plasmodium falciparum en Córdoba, Colombia
}

\author{
Angélica Knudson ${ }^{1,2}$, Rubén Santiago Nicholls ${ }^{1}$, Ángela Patricia Guerra ${ }^{3}$, Ricardo Sánchez ${ }^{4}$ \\ ${ }^{1}$ Grupo de Parasitología, Subdirección de Investigación, Instituto Nacional de Salud, Bogotá, D.C., \\ Colombia \\ 2 Departamento de Microbiología, Facultad de Medicina, Universidad Nacional de Colombia, Bogotá,D.C., \\ Colombia \\ ${ }^{3}$ Grupo de Bioquímica y Biología Celular, Subdirección de Investigación, Instituto Nacional de Salud, \\ Bogotá, D.C., Colombia \\ ${ }^{4}$ Departamento de Psiquiatría, Facultad de Medicina, Universidad Nacional de Colombia, Bogotá, D.C. \\ Colombia
}

Introducción. Pocos estudios describen el conjunto de características clínicas de los pacientes con paludismo no complicado por Plasmodium falciparum en Córdoba, Colombia.

Objetivo. Describir diferentes perfiles de pacientes con paludismo no complicado por $P$. falciparum, tratados con cloroquina, en Tierralta y Puerto Libertador, Córdoba, a partir de sus variables clínicas, epidemiológicas y de laboratorio.

Materiales y métodos. Se evaluaron pacientes con paludismo no complicado por $P$. falciparum según los protocolos estandarizados por la Organización Panamericana de la Salud y se recolectó información clínica y parasitológica. Se utilizó análisis multivariado por correspondencias múltiples para describir diferentes perfiles de pacientes con paludismo no complicado por $P$. falciparum, tratados con cloroquina.

Resultados. Se evaluaron 127 pacientes, 105 de ellos con seguimiento completo por 14 días; $6,7 \%$ presentó respuesta clínica adecuada. Entre $80 \%$ y $98 \%$ de los pacientes refirió, por lo menos, uno de los síntomas más frecuentes del cuadro clínico habitual del paludismo no complicado y $80,3 \%$ presentó decaimiento como signo clínico más frecuentemente encontrado. El análisis multivariado permitió agrupar las variables en cinco perfiles distintos de presentaciones clínicas que se describen en detalle.

Conclusiones. Existe una alta frecuencia $(93,3 \%)$ de fallas a la cloroquina como tratamiento para el paludismo no complicado por $P$. falciparum en esta región. Las agrupaciones hechas con el análisis por correspondencias múltiples mostraron similitudes con las descripciones clásicas encontradas en la literatura sobre las formas de presentación clínica de la malaria no complicada. La baja frecuencia de individuos con respuesta clínica adecuada impidió el análisis de asociación. El análisis multivariado involucra variables relacionadas con aspectos epidemiológicos y clínicos y permite una interpretación más integral de los hallazgos obtenidos.

Palabras clave: malaria, Plasmodium falciparum, cloroquina, análisis multivariado, síntomas clínicos.

Clinical profiles of patients with uncomplicated Plasmodum falciparum malaria in northwestern Colombia

Introduction. Few studies describe the clinical presentations of uncomplicated Plasmodium falciparum malaria in the province of Córdoba in an endemic area of northwestern Colombia. Objective. Profiles of patients with uncomplicated Plasmodium falciparum malaria were described from two twons of Córdoba, Tierrata and Puerto Libertador, based on clinical, epidemiological and laboratory variables.

Materials and methods. Patients were examined according to standard WHO/PAHO protocols for assessment of antimalarial drug efficacy. Clinical data and parasitological information was collected as well. A multiple correspondence multivariate analysis was used to compare the profiles of 127 patients with uncomplicated Plasmodium falciparum malaria. 
Results. Of the 127patients, 105 completed the 14-day follow-up and 7 had adequate clinical response. Between $80 \%$ and $98 \%$ of patients exhibited at least one of the most frequent symptoms of uncomplicated malaria, and $80.3 \%$ had asthenia as the most frequent symptom. The multivariate analysis grouped the variables into five distinguishable clusters of clinical profiles. These groups showed similarities with the classical clinical descriptions of uncomplicated malaria encountered in the literature. The low frequency of patients with adequate clinical response hampered the association analysis.

Conclusions. In Córdoba, therapeutic failure to chloroquine treatment is high in treating uncomplicated Plasmodium falciparum malaria. Multivariate analysis summarized variables related to epidemiological and clinical aspects and permitted a more objective approach to the interpretation of the findings.

Key words: malaria, Plasmodium falciparum, chloroquine, multivariate analysis, symptoms, clinical.

El paludismo es una enfermedad parasitaria febril aguda cuya gravedad y evolución dependen de muchos factores, como la especie del parásito causante de la infección, el origen geográfico de la infección y factores propios del huésped como la edad, la constitución genética, los estados inmunitario, nutricional y de salud del paciente, además de la respuesta a la quimioterapia utilizada (1).

La infección por Plasmodium falciparum tiene un amplio espectro de manifestaciones clínicas que pueden ir desde la forma asintomática, pasando por la malaria no complicada, hasta cuadros clínicos complicados con desenlace fatal (2).

La expresión habitual del paludismo no complicado se caracteriza por escalofrío, fiebre y sudoración, acompañados de cefalea, náuseas y, con menor frecuencia, dolor abdominal, vómito y diarrea. En los casos graves se pueden encontrar anemia, hepatoesplenomegalia, compromiso cerebral, falla renal y choque. Las complicaciones pueden deteriorar gravemente el estado de salud del individuo y conducir a la muerte (3).

Las manifestaciones clínicas de la malaria en zonas de alta transmisión son más frecuentes en niños y jóvenes, mientras que las infecciones asintomáticas son más comunes en individuos mayores. Esto indica que la inmunidad es adquirida

\footnotetext{
Correspondencia:

Angélica Knudson, Departamento de Microbiología, Facultad de Medicina, Universidad Nacional de Colombia, Ciudad Universitaria, carrera 30 calle 45 , edificio 471 , tercer piso, oficina 304A, Bogotá, D.C.

Teléfono: (057) 316 5000, extensión 15016

raknudsono@unal.edu.co
}

Recibido: 21/09/06; aceptado: 20/09/07 gradualmente y está relacionada con la edad en la que ocurre la primera exposición, ya que los niños no alcanzan a desarrollar mecanismos inmunes cuando entran en contacto con el parásito, aumentando el riesgo de adquirir paludismo clínicamente manifiesto (4). Los pacientes no inmunes con infecciones causadas por $P$. falciparum pueden presentar parasitemias elevadas y síntomas intensos a menos que sean diagnosticados y tratados oportunamente. En regiones donde la malaria es endémica, en las cuales los individuos tienen algún grado de inmunidad, las infecciones graves son poco comunes $(4,5)$; sólo una pequeña proporción de infecciones puede alcanzar una parasitemia elevada que conduzca a un cuadro complicado de la enfermedad (5).

Debido a su bajo costo, seguridad y efectividad, la cloroquina fue el medicamento de elección para el tratamiento del paludismo no complicado causado por $P$. falciparum en muchas regiones del mundo, aunque hace más de 40 años emergió la resistencia a este medicamento en Asia y Suramérica, que empeoró la morbimortalidad por esta entidad en el mundo (6).

En Colombia, la cloroquina se recomendó, sola o en combinación con sulfadoxina-pirimetamina, como esquema de primera línea para el tratamiento del paludismo no complicado causado por $P$. falciparum desde la década de 1950 hasta finales de la década de 1990. En Colombia, desde 1959 se han informado casos de falla terapéutica del tratamiento con cloroquina según diferentes metodologías $(7,8)$ y desde 1998 , con los protocolos estandarizados por la Organización Mundial 
de la Salud y la Organización Panamericana de la Salud (OMS/OPS) (9) se han informado niveles de falla entre $44 \%$ y $97 \%(10-13)$.

A partir del año 2000, el Ministerio de Salud modificó el esquema de tratamiento de primera línea del paludismo no complicado causado por $P$. falciparum, por la combinación de amodiaquina más sulfadoxina-pirimetamina (14). A pesar de ello, en muchas áreas endémicas del país se continuó utilizando cloroquina, sola o en combinación con sulfadoxina-pirimetamina, situación que se presentó hasta finales de 2003 , debido a dificultades para la obtención de amodiaquina en cantidades suficientes para suplir la demanda nacional.

El adecuado conocimiento de la presentación de las manifestaciones clínicas del paludismo no complicado en pacientes ambulatorios provenientes de zonas endémicas de baja transmisión y su relación con la respuesta al tratamiento antipalúdico, contribuye a mejorar la orientación de las estrategias encaminadas al control de esta enfermedad y a su adecuada evaluación. Existen muy pocos estudios que describan la presentación clínica de la malaria y su respuesta al tratamiento en las diferentes zonas endémicas en Colombia, la mayoría de los cuales están encaminados al análisis de los hallazgos clínicos y de laboratorio en los casos de paludismo complicado $(15,16)$.

El presente estudio tuvo como objetivo describir diferentes perfiles de pacientes con paludismo no complicado por $P$. falciparum, tratados con cloroquina, en Tierralta y Puerto Libertador, Córdoba, a partir de sus variables clínicas, epidemiológicas y de laboratorio (parasitemia) utilizando como método de análisis la evaluación por correspondencias múltiples aunque sin el propósito de predecir el desenlace de la respuesta. A pesar de que se tenía información acerca de fallas al tratamiento con cloroquina en Antioquia y en la Costa Pacífica, no había información de estudios realizados en Córdoba.

\section{Materiales y métodos}

\section{Sitio de estudio}

Los pacientes provenían de los municipios de Tierralta (latitud norte: $08^{\circ} 10^{\prime} 34^{\prime \prime}$, longitud oeste: $76^{\circ} 03^{\prime} 46^{\prime \prime}$ ) y Puerto Libertador (latitud norte: $07^{\circ}$
53' 53", longitud oeste: $\left.75^{\circ} 40^{\prime} 25 "\right)$, Córdoba, ubicados en la zona del Bajo Cauca-Alto Sinú, considerada una región de alto riesgo de transmisión para malaria en Colombia por presentar índices parasitarios anuales mayores de $10(17,18)$. El estudio se realizó entre noviembre de 2002 y julio de 2003.

\section{Selección de los pacientes}

Se siguió la metodología propuesta en los protocolos estandarizados por la OMS y la OPS para evaluación de la eficacia de la cloroquina para el tratamiento del paludismo no complicado causado por $P$. falciparum en áreas de baja transmisión (9), con algunas modificaciones, las cuales fueron: inclusión de pacientes mayores de dos años de edad y recuento parasitario entre 450 y 5.000 formas asexuales de $P$. falciparum/ $\mu$ lde sangre. Se recolectó información sobre variables demográficas, clínicas y de laboratorio de los pacientes incluidos en el estudio.

Al ingresar al estudio se aplicó un instrumento que indagaba sobre antecedentes y síntomas relacionados con la presentación clínica del paludismo no complicado y se consignaron datos sobre hallazgos clínicos durante el examen físico practicado por un médico a cada paciente. Se realizó seguimiento clínico y parasitológico en los días $0,1,2,3,7$ y 14 . A los pacientes se les administró tratamiento supervisado con cloroquina en dosis total de $25 \mathrm{mg} / \mathrm{kg}$ de peso, repartida en tres días con dosis diarias de $10,7,5$ y $7,5 \mathrm{mg} / \mathrm{kg}$. Como tratamiento de rescate se utilizó la combinación amodiaquina, $25 \mathrm{mg} / \mathrm{kg}$, más sulfadoxina-pirimetamina, $25 \mathrm{mg} / \mathrm{kg}-1,25 \mathrm{mg} / \mathrm{kg}$.

La respuesta terapéutica y el desenlace final fueron clasificados como falla terapéutica precoz, falla terapéutica tardía, respuesta clínica adecuada, deserción y exclusión de acuerdo con las definiciones establecidas en el protocolo de OMS/ OPS (9). La respuesta parasitológica se clasificó como RI temprana, RI tardía, RII, RIII y sensible conforme con las definiciones de OMS/OPS (9).

\section{Diagnóstico parasitológico}

El diagnóstico parasitológico se llevó a cabo en muestras de gota gruesa y extendidos de sangre periférica teñidos con la coloración de 
Romanowsky modificada (19). Las muestras fueron observadas con el microscopio de luz, con aumento de 1.000X. El recuento de las formas parasitarias se realizó en 200 leucocitos con constante de 8.000 leucocitos/ $\mu$ l de sangre (9).

\section{Análisis estadístico}

Se realizó un análisis descriptivo de las variables demográficas, clínicas y de laboratorio con las medidas convencionales (proporciones, medias, medianas, medidas de dispersión), según las características de cada variable. Los estimadores fueron calculados junto con sus respectivos intervalos de confianza del 95\% (IC95\%). Los anteriores procedimientos fueron ejecutados con el programa Stata $8 \AA$.

El análisis estadístico de los datos tuvo en cuenta el propósito de estudiar asociación entre variables fundamentalmente de tipo categórico, razón por la que se utilizó un método de análisis multivariado, el de correspondencias múltiples. Con este método se pueden analizar los perfiles configurados por múltiples variables en términos de semejanza o proximidad de las diferentes modalidades (20), lo cual permite identificar grupos con significación clínica a partir de diferentes combinaciones de categorías de variables, e incorporar dentro de estos grupos el comportamiento de variables continuas. Los perfiles pueden analizarse en una representación espacial bidimensional en la cual es posible ubicar las diferentes modalidades de las variables en un plano cartesiano con un polo positivo y uno negativo. Para dicho análisis se seleccionan dos grupos de variables: variables activas, que son las que participan en la construcción de los ejes factoriales, y variables suplementarias o ilustrativas, que son variables que permiten enriquecer la interpretación de los ejes factoriales, una vez éstos se han construido (21).

En el análisis multivariado fueron tratadas como activas las siguientes variables, relacionadas con características clínicas, de tratamiento y de antecedentes: sexo, escalofríos, sudoración, dolor osteomuscular, dolor abdominal, anorexia, náuseas, vómito, astenia, adinamia, palidez conjuntival, palidez palmar, hepatomegalia y esplenomegalia, uso de cloroquina, otros antipalúdicos o acetaminofén en el episodio actual y antecedente de paludismo en el último año. Se tomaron como variables ilustrativas la falla terapéutica y las variables cuantitativas (edad, días de enfermedad, temperatura, número de formas asexuales de $P$. falciparum). En las figuras se muestra cómo se proyectan sobre cada uno de los factores las variables activas, representadas por cuadrados rellenos de diferente tamaño, las nominales ilustrativas, señaladas con cuadros vacíos, y las continuas ilustrativas, representadas por flechas. El tamaño de los cuadros es proporcional a la contribución de cada modalidad sobre el factor (eje factorial) más representativo. Las figuras permiten ver las agrupaciones de variables activas que conforman los diferentes perfiles $y$, además, permiten analizar la estructura de dichos perfiles sobre una representación bidimensional. La correlación de las variables continuas ilustrativas con cada uno de los factores corresponde a la longitud de la proyección de la flecha sobre el factor respectivo. Los procedimientos estadísticos se efectuaron con el paquete estadístico SPAD $4 \AA$.

\section{Aspectos éticos}

El proyecto fue aprobado por el Comité de Ética del Instituto Nacional de Salud y presentado, discutido y aprobado por parte de las autoridades departamentales de salud de los sitios en donde se realizó el trabajo de campo. Todos los pacientes fueron informados acerca del estudio y quienes accedieron a participar, firmaron el formato de consentimiento informado. En el caso de los menores de edad, el consentimiento fue firmado por sus padres o tutores.

Previendo la posibilidad de que pudiera presentarse un alto porcentaje de fallas terapéuticas al tratamiento con cloroquina, con el fin de evitar el desarrollo de posibles complicaciones se estableció como criterio de inclusión que la parasitemia asexual en el día 0 no fuera mayor de 5.000 parásitos/ $/$ l. Además de ello, se tomaron todas las previsiones necesarias con el fin de garantizar que los pacientes no se perdieran durante los días establecidos en el protocolo para las evaluaciones clínicas o parasitológicas, incluyendo la 
realización de visitas domiciliarias siempre que se consideró necesario, con el fin de garantizar siempre la protección de los pacientes incluidos en el estudio.

\section{Resultados}

Se evaluaron 127 pacientes (59 en Tierralta y 68 en Puerto Libertador); 105 de ellos tuvieron seguimiento completo; $17,3 \%(22 / 127)$ de los pacientes no terminó el seguimiento; $12,6 \%$ (16/ 127) fueron desertores después de haber sido incluidos en el estudio y $4,7 \%$ (6/127) fueron excluidos del estudio durante el seguimiento por diferentes motivos, entre ellos, la presencia de una enfermedad concomitante que dificultó la clasificación clara del resultado del tratamiento, el traslado del paciente a un lugar fuera del alcance del seguimiento activo y el retiro voluntario.

La media de la edad fue de $27,5 \pm 15,6$, con rango entre 2 y 68 años. El $50 \%$ de los pacientes eran mayores de 25 años y $77,17 \%$ (98/127) eran hombres (IC95\%: 62,7\% a 94,0\%).

Los 127 pacientes evaluados al inicio del estudio presentaron una mediana de un episodio de malaria en el último año previo al episodio actual, con un rango intercuartil entre 0 y 2 . De estos pacientes, $51,2 \%$ (IC95\%: 39,5\% a 65,2\%) presentó, por lo menos, un episodio de paludismo en el año previo al episodio actual. El promedio de tiempo de enfermedad antes de la consulta fue de 5,4 días, con un rango entre 1 y 30 días y una desviación estándar de 5,1.

En total, $82,7 \%$ de los 127 pacientes (IC95\%: $67,6 \%$ a $100 \%$ ) dijo no haber recibido cloroquina antes de confirmar el diagnóstico y $86,6 \%$ (IC95\%: $71,1 \%$ a $100 \%$ ) dijo no haber recibido ningún otro antipalúdico, excepto por 0,8\% (IC95\%: 0,02\% a $4,3 \%)$ que recibió sulfadoxina-pirimetamina y primaquina y $7,9 \%$ (IC95\%: 3,8\% a 14,5\%) recibió sólo primaquina. El 37\% de los 127 pacientes (IC95\%: 27,2\% a 49,2\%) recibió acetaminofén antes de la consulta para el control de los síntomas del paludismo.

A los 127 pacientes evaluados al inicio del estudio se les indagó sobre síntomas relacionados con la presentación clínica de la malaria en el día cero y se realizó su evaluación clínica y parasitológica en los días recomendados en el protocolo. Los síntomas que se presentaron con mayor frecuencia en el día de ingreso fueron fiebre, escalofrío y sudoración. El signo más frecuente fue decaimiento (80,3\% [IC95\%: 65,5 a 97,5]), seguido de palidez conjuntival (54,33\% [IC95\%: $42,3$ a 68,8$]$ ) y de hepatomegalia o esplenomegalia (45,7\% [IC95\%: 34,7 a 59]). La frecuencia de los hallazgos clínicos se presenta en el cuadro 1.

Al ingresar al estudio, 91,3\% de los 127 pacientes evaluados (IC95\%: $75,5 \%$ a $100 \%$ ) no tenían gametocitemia en la gota gruesa.

De los 105 pacientes con seguimiento completo a 14 días, 54,3\% (57/105) (IC95\%: 41,1\% a 70,3\%) presentaron falla terapéutica precoz, 39\% (41/105) (IC95\%: 28\% a 53\%) presentaron falla terapéutica tardía y 6,7\% (7/105) (IC95\%: 2,7\% a 13,7\%) presentaron respuesta clínica adecuada. La respuesta parasitológica tipo RIII se presentó en 61,9\% (65/ 105) (IC95\%: 47,78 a 78,9) de estos pacientes. En relación con los días de seguimiento 35,7\% (35/98) (IC95\%: 24,9\% a 49,7\%) presentaron falla terapéutica en el día siete. La presencia de $P$. falciparum en los controles de los días 7 y 14 como razón de falla del tratamiento, se encontró en 38,10\% (40/105) (IC95\%: 27,22 a 51,87) de los casos. Estos datos se resumen en el cuadro 2.

En el análisis multivariado con las técnicas de correspondencias múltiples, mediante el análisis del cambio de inercia en un gráfico de sedimentación de valores propios, se identificaron cinco factores (ejes factoriales) que permitieron agrupar las variables en perfiles descritos a continuación, las cuales se resumen en el cuadro 3.

\section{Perfil 1: síntomas clínicos frecuentes y su relación con falla terapéutica}

En esta agrupación la presencia de síntomas clínicos (fiebre, escalofrío, palidez palmar, hepatomegalia y síntomas gastrointestinales) se asoció con la falla del tratamiento con cloroquina y con cifras de temperatura axilar mayores; este grupo incorporó el uso previo de primaquina como un factor asociado a la falla terapéutica. Es característico de este perfil que a mayor edad se tendía a presentar menos sintomatología y menos falla del tratamiento con cloroquina (figura 1). 
Cuadro 1. Frecuencia de presentación de los síntomas y signos evaluados en el día del ingreso al estudio (día 0), con sus intervalos de confianza del $95 \%$.

\begin{tabular}{|c|c|c|c|c|}
\hline Síntoma indagado & & $\begin{array}{l}0(\%) \\
127)\end{array}$ & \multicolumn{2}{|c|}{$\mathrm{IC} 95 \%$} \\
\hline $\begin{array}{l}\text { Fiebre } \\
\text { Escalofrío } \\
\text { Sudoración } \\
\text { Dolor osteomuscular } \\
\text { Dolor abdominal } \\
\text { Anorexia } \\
\text { Náuseas } \\
\text { Vómito }\end{array}$ & & $\begin{array}{r}3,43 \\
3,19 \\
, 31 \\
5,59 \\
3,03 \\
5,59 \\
2,20 \\
, 56\end{array}$ & $\begin{array}{l}81,93 \mathrm{a} \\
72,61 \mathrm{a} \\
65,49 \mathrm{a} \\
61,23 \mathrm{a} \\
36,74 \mathrm{a} \\
61,23 \mathrm{a} \\
49,25 \mathrm{a} \\
19,20 \mathrm{a}\end{array}$ & \\
\hline $\begin{array}{l}\text { Signos evaluados } \\
\text { Paciente decaído } \\
\text { Palidez conjuntival } \\
\text { Palidez palmar } \\
\text { Hepatomegalia } \\
\text { Esplenomegalia }\end{array}$ & & $\begin{array}{r}0,31 \\
+, 33 \\
5,43 \\
0,22 \\
9,92\end{array}$ & $\begin{array}{l}65,49 \text { a } \\
42,27 \text { a } 6 \\
25,85 \text { a } \\
26,52 \text { a } \\
21,18 \text { a }\end{array}$ & \\
\hline $\begin{array}{l}\text { Temperatuta axilar }\left({ }^{\circ} \mathrm{C}\right) \\
\text { Parasitemia asexual } \\
\text { (parásitos } / \mu \text { de sangre) }\end{array}$ & $\begin{array}{c}\text { Media } \\
37,13 \\
2018,24\end{array}$ & $\begin{array}{c}\text { Rango } \\
36 \text { a } 39,4 \\
500 \text { a } 5000\end{array}$ & $\begin{array}{c}\text { Desviación estándar } \\
0,9 \\
1269,31\end{array}$ & $\begin{array}{c}\text { Mediana } \\
37 \\
1743\end{array}$ \\
\hline $\begin{array}{l}\text { Parasitemia sexual } \\
\text { (gametocitos/ } / \mu \text { de sangre) }\end{array}$ & 24,06 & 0 a 816 & 111,50 & 0 \\
\hline
\end{tabular}

\section{Perfil 2: características clínicas y pronósticas en relación con el tiempo de evolución de la sintomatología}

Agrupó a los pacientes en relación con el tiempo de evolución de la enfermedad: aquéllos con sintomatología más aguda (menor tiempo de evolución) tenían la tendencia de presentar más fiebre, sudoración, astenia y hepatomegalia. Los pacientes que presentaron mayor tiempo de evolución de la enfermedad presentaron más palidez, tanto palmar como conjuntival, y dolor abdominal; además, fueron propensos a presentar más fallas tardías con el tratamiento con cloroquina y a tener antecedente de episodios de paludismo en el último año (figuras 1 y 2).

Perfil 3: evolución aguda con síntomas de hemólisis en pacientes sin tratamiento previo

Este perfil permitió diferenciar a los pacientes que presentaron sintomatología de tipo hepatoesplenomegalia y palidez, signos compatibles con hemólisis, menos tiempo de duración de la enfermedad, mayor parasitemia asexual y no estuvieron expuestos a los antipalúdicos, en contraste con pacientes con mayor duración de la enfermedad, que tenían la tendencia de presentar sintomatología menos Ilamativa (solamente sudoración y escalofrío), asociada al uso previo de antipalúdicos, como la primaquina y la cloroquina; este grupo de pacientes también mostró menor parasitemia asexual (figura 2).

\section{Perfil 4: intensidad de síntomas, tratamiento y variables demográficas}

Agrupó a los pacientes en relación con el sexo y la edad: los hombres de mayor edad presentaron sintomatología más intensa (hepatomegalia y esplenomegalia con dolor abdominal y osteomuscular), mientras que las mujeres jóvenes, aunque tuvieron mayor presencia de formas asexuales en el examen parasitológico, sólo presentaron vómito y palidez. En el grupo de hombres se presentó consumo previo de antipalúdicos antes del diagnóstico, como la cloroquina, mientras que en las mujeres se halló uso de acetaminofén (figura 3 ). 
Cuadro 2. Distribución de casos según día de falla terapéutica, tipo de respuesta parasitológica y razón de la falla.

Los denominadores para el análisis del día de la falla corresponden a los 98 pacientes que presentaron falla terapéutica al tratamiento con cloroquina. En los casos del tipo de respuesta parasitológica y la razón de falla, los denominadores incluyen tanto a las fallas como a las respuestas clínicas adecuadas.

- Día 1

- Día 2

- Día 3

- Día 7

- Día 14

Respuesta parasitológica

- RI temprana

- RII

- RIII

- Sensible

- No clasificables*

Razón de falla

- Presencia de algún signo de peligro o paludismo grave en los días 1 o 2

- Mayor parasitemia asexual el día 2 que el día 0

- Parasitemia asexual el día 3 igual o mayor que $25 \%$ de la parasitema del día 0

- Presencia de signos de peligro o paludismo grave después del día 3 , con parasitemia de la misma especie que el día 0

- Presencia de parasitemia de la misma especie que el día 0 , en los controles programados para los días 7 y 14

- Respuesta clínica adecuada

\begin{tabular}{|c|c|}
\hline $4,1(4 / 98)$ & 1,11 a 10,45 \\
\hline $26,5(26 / 98)$ & 17,34 a 38,87 \\
\hline $27,6(27 / 98)$ & 18,16 a 40,08 \\
\hline $35,7(35 / 98)$ & 24,88 a 49,67 \\
\hline $6,1(6 / 98)$ & 2,25 a 13,32 \\
\hline
\end{tabular}

$4,76(5 / 105)$

1,55 a 11,11

13,13 a 31,72

47,78 a 78,9

2,68 a 13,73

2,1 a 12,44

$5,71(6 / 105)$

2,1 a 12,44

$22,86(24 / 105)$

14,65 a 34,01

$25,71(27 / 105)$

16,95 a 37,41

$0,95(1 / 105)$

0,02 a 5,31

$38,10(40 / 105)$

27,22 a 51,87

\begin{abstract}
* Pacientes que no cumplieron con los requisitos para ser
consignadas en los protocolos de la OMS/OPS.
Perfil 5: síntomas agudos clásicos de
paludismo y su relación con el tiempo de la paludismo y su relación
enfermedad y la edad
\end{abstract}

En esta agrupación los pacientes con mayor edad pero corto tiempo de evolución de la enfermedad, presentaron sintomatología florida, propia de un episodio agudo de malaria (sudoración, escalofrío, anorexia y dolor abdominal y osteomuscular), en contratse con los pacientes jóvenes con mayor tiempo de evolución, que usaron antipalúdicos, como la primaquina y la cloroquina, y tuvieron más signos que síntomas (hepatomegalia y esplenomegalia). Al contrastar este perfil con el número 1 (síntomas clínicos frecuentes y su relación con falla terapéutica) se encontró que el tiempo de evolución de la enfermedad en jóvenes pudo ser un factor relacionado con la menor gravedad de la sintomatología (figura 3).

\section{Discusión}

Los pacientes evaluados correspondieron a casos de paludismo no complicado causado por $P$. falciparum, quienes presentaron con mayor frecuencia la tríada clásica de sintomatología de esta enfermedad: escalofrío, fiebre y sudoración. Los pacientes tuvieron baja exposición a la infección pues a pesar de residir en áreas de transmisión de malaria en el país, en las cuales se registran habitualmente índices parasitarios anuales superiores a 10 por $1.000(17,18)$, para casi la mitad de ellos fue el primer episodio de paludismo en el año previo al momento de la consulta. En contraste, en zonas de alta y 
Cuadro 3. Estructuras de los perfiles encontrados por medio del análisis multivariado. Las variables ilustrativas se señalan en negrilla y cursiva. Su ubicación en el polo del eje depende del sentido de su proyección, la cual se muestra en cada figura.

\begin{tabular}{lll}
\hline Perfil & Polo negativo & Polo positivo \\
\hline
\end{tabular}

1. Síntomas clínicos frecuentes y su relación con falla terapéutica

2. Características clínicas y pronósticas en relación con el tiempo de evolución de la sintomatología

3. Evolución aguda con síntomas de hemólisis en pacientes sin previo tratamiento

4. Intensidad de síntomas, tratamiento y variables demográficas

5. Síntomas agudos clásicos de paludismo y su relación con el tiempo de la enfermedad y la edad
Temperatura axilar

Presencia de falla terapéutica

Presencia de escalofrío, palidez palmar, hepatomegalia, síntomas gastrointestinales Antecedente de uso de acetaminofén Antecedente de uso previo de antipalúdicos tipo primaquina

Días de enfermedad

Presencia de palidez palmar

y conjuntival, dolor abdominal,

fallas tardías con el tratamiento con cloroquina

Antecedente de episodio

de paludismo en el último año

Días de enfermedad

Presencia de sudoración, escalofrío

Antecedentes de uso previo

de antipalúdicos como la primaquina

y la cloroquina

Edad

Presencia de hepatoesplenomegalia, dolor abdominal y osteomuscular

Sexo masculino

Antecedente de uso de antipalúdicos previo al diagnóstico, como la cloroquina

Edad

Presencia de sudoración,

escalofrío, anorexia,

dolor abdominal y osteomuscular
Ausencia de falla terapéutica

Edad

Ausencia de hepatoesplenomegalia,

síntomas gastrointestinales,

palidez palmar

Sin antecedente previo de uso de acetaminofén

Temperatura axilar

Presencia de sudoración, astenia,

hepatomegalia

Sin antecedentes de episodio de paludismo en el último año

Formas asexuales de P. falciparum Presencia de hepatoesplenomegalia, palidez palmar y conjuntival

Sin antecedente de uso previo de antipalúdicos

Formas asexuales de P. falciparum

Presencia de vómito, palidez conjuntival

Sexo femenino

Antecedentes de uso de acetaminofén

Días de enfermedad

Antecedentes de uso de

antipalúdicos previo al

diagnóstico, como la

la primaquina y la cloroquina

Presencia de hepatoesplenomegalia permanente transmisión en otras regiones del mundo, como en Dielmo, Senegal, el promedio de episodios de paludismo al año puede ser de seis en población menor de dos años, con una reducción de la frecuencia de ellos entre los 10 y los 15 años de edad, hasta presentar una episodio cada siete años en la población adulta (22).

El $80 \%$ de los pacientes presentó decaimiento como signo principal y $45,7 \%$ tuvo hepatomegalia o esplenomegalia. A pesar de que $98 \%$ de los pacientes refirieron haber tenido fiebre en las 24 horas previas al momento de la consulta, el promedio de temperatura al ingreso fue de $37,1^{\circ} \mathrm{C}$, aunque se registraron temperaturas hasta de $39,4^{\circ} \mathrm{C}$ en algunos casos. La fiebre es un síntoma importante entre las manifestaciones clínicas de la malaria y en áreas de transmisión estacional, como en Madagascar, la parasitemia asintomática no es común (23), lo que podría ocurrir también en Colombia (24). Sin embargo, se debe recordar que el paludismo puede no ser la única causa de fiebre, aun cuando se encuentre una gota gruesa positiva para Plasmodium spp. Por tal motivo, se recomienda adoptar definiciones operativas en los casos de paludismo no complicado con el fin de tener una buena caracterización de la presentación clínica, en la cual se contemplen otras variables clínicas y desarrollar el concepto de umbral pirogénico, que ha sido aplicado por algunos autores en diversos estudios de este tipo (23). 


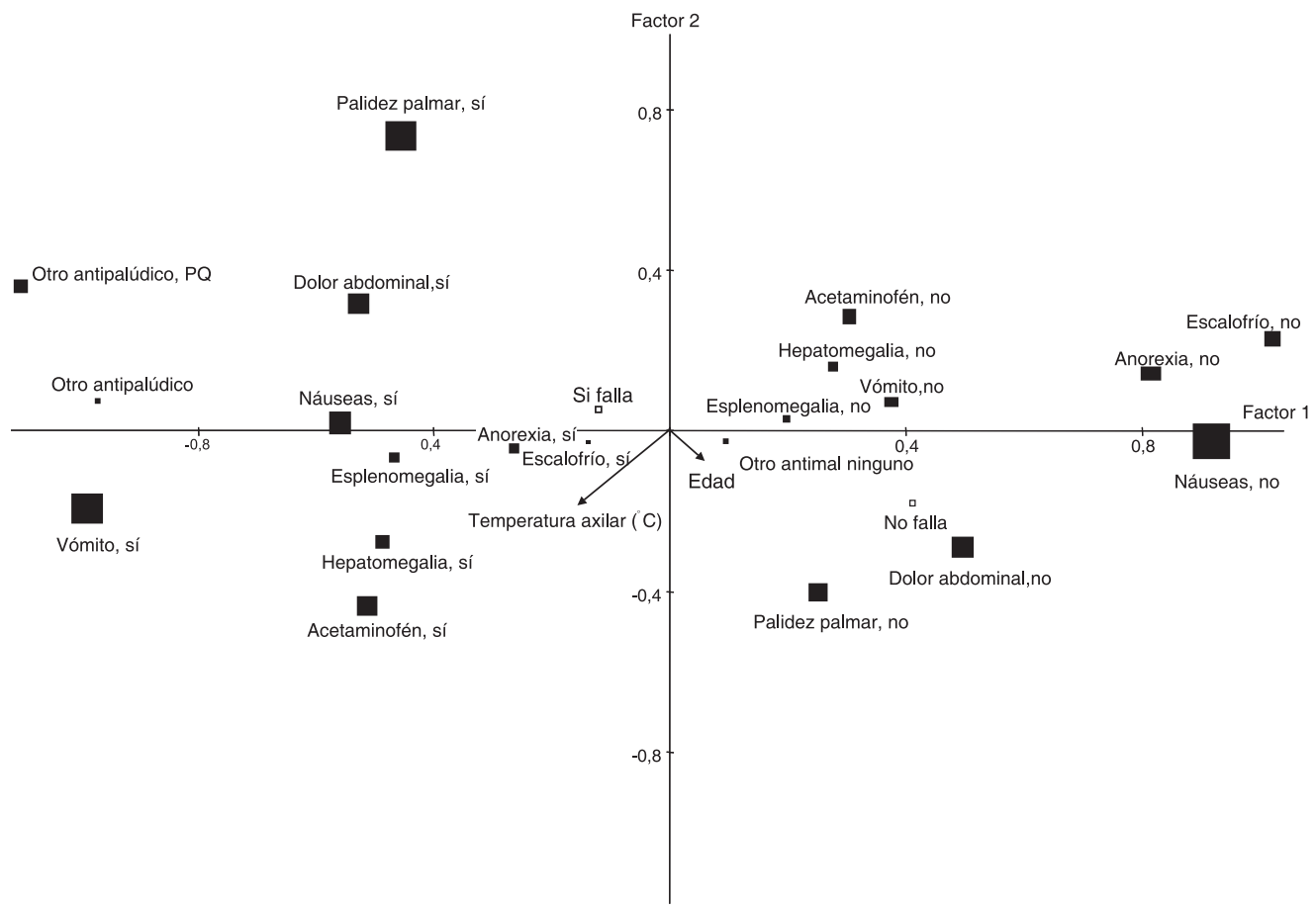

Figura 1. Representación de las modalidades sobre los dos primeros factores.

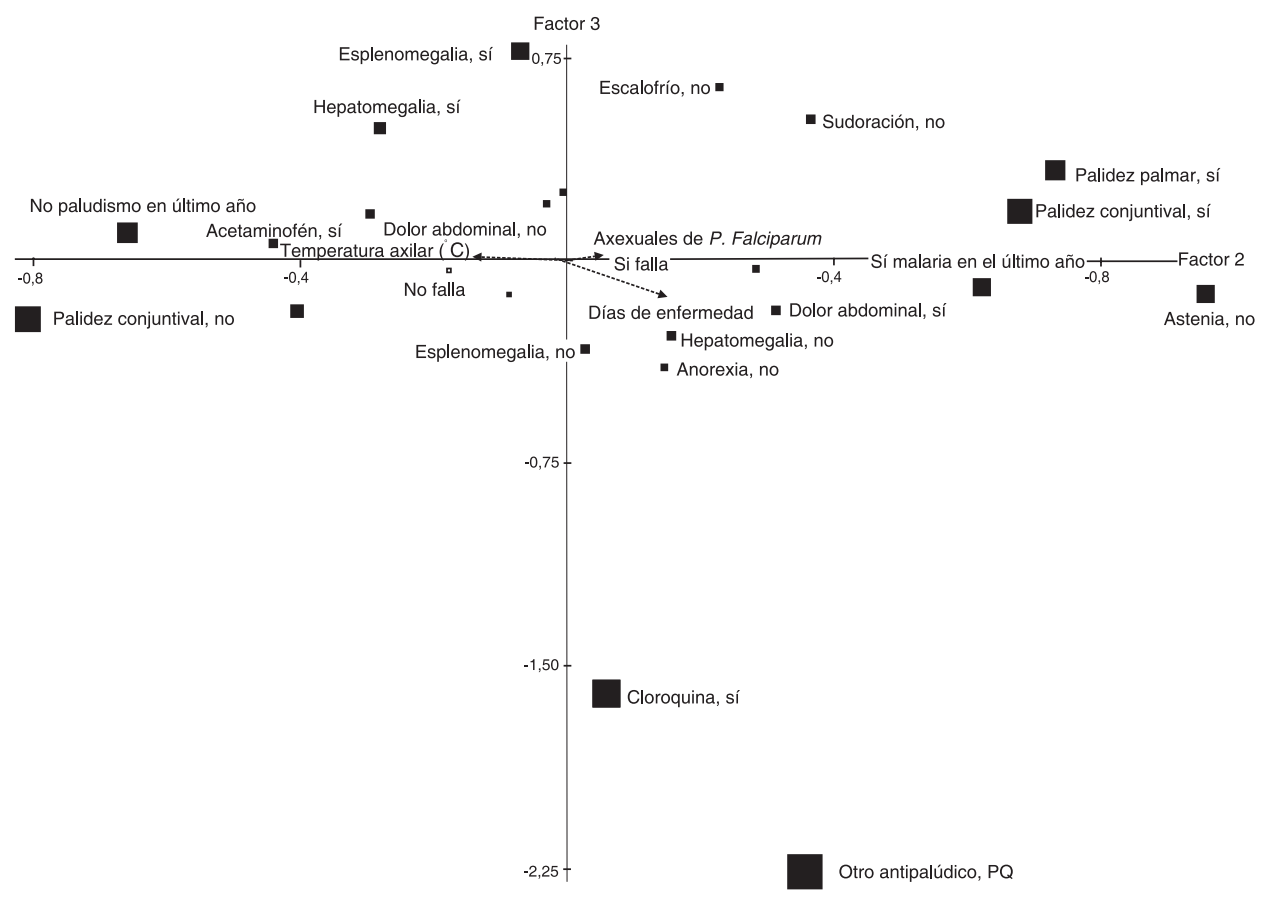

Figura 2. Representación de las modalidades sobre los factores dos y tres. 


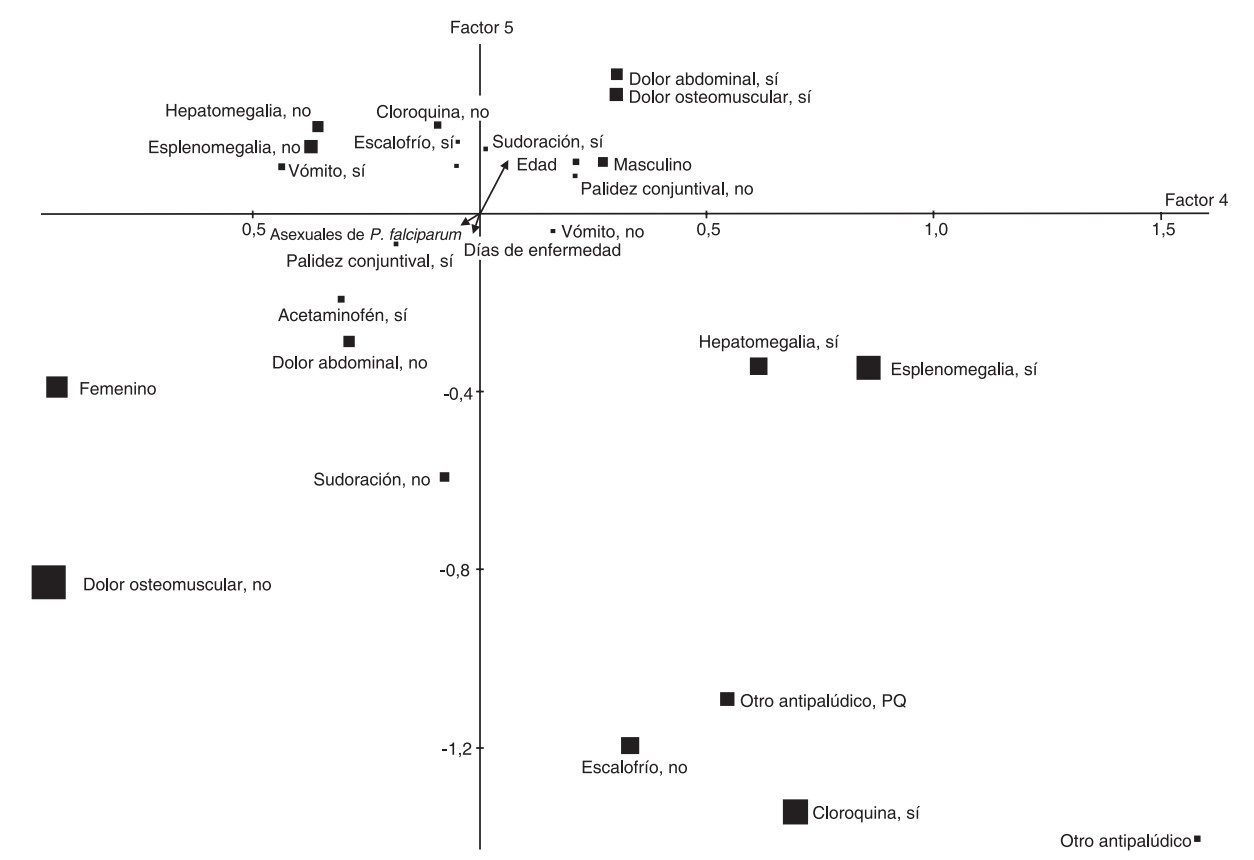

Figura 3. Representación de las modalidades sobre los factores cuatro y cinco.

En relación con las intervenciones terapéuticas estudiadas, se encontró que $37 \%$ de los pacientes usó acetaminofén para el tratamiento de los síntomas asociados, como la fiebre. El uso de medicamentos antipalúdicos automedicados se presentó en $0,8 \%$ a $17,3 \%$ de los pacientes, lo cual no es tan frecuente como se esperaría en regiones donde el acceso oportuno al diagnóstico y tratamiento de la malaria, factor que contribuye a determinar la respuesta terapéutica, es deficiente. En poblaciones en las que es frecuente el uso popular inadecuado a dosis subterapéuticas o como monoterapia de los medicamentos antipalúdicos, se promueve el desarrollo de resistencia parasitaria (25) y se podría prolongar la duración del cuadro clínico y empeorar las manifestaciones del mismo.

El método de análisis multivariado identificó varios aspectos para destacar. En el perfil 1 la relación de los pacientes con mayor edad, menor frecuencia de sintomatología y menor frecuencia de falla del tratamiento con cloroquina, podría ser explicada por el desarrollo, a medida que avanza la edad, de respuesta inmune con cierto grado de protección contra el parásito adquirida luego de frecuentes exposiciones del individuo a lo largo de su vida (26). Los pacientes agrupados en el perfil 2 característicamente presentaron un cuadro de sintomatología febril asociada a un tiempo corto de evolución de la enfermedad, lo cual se encuentra frecuentemente en zonas de transmisión baja e inestable, como en Colombia (27).

También se hallaron pacientes con sintomatología compatible con hemólisis (palidez mucocutánea y hepatoesplenomegalia) (perfil 3) a quienes, además, se les encontró mayor parasitemia asexual. La anemia en el paludismo es de origen multifactorial, debida a la destrucción esplénica de los hematíes parasitados, a la diseritropoyesis, ocasionalmente a la pérdida sanguínea por sangrado, y a la existencia de hemólisis de tipo inmune (26). El proceso de destrucción de eritrocitos por el bazo se lleva a cabo durante un período de varias semanas después de la infección aguda (26), proceso más intenso en pacientes con esplenomegalia (28).

En el perfil 4 se agruparon hombres adultos con presentación clínica más grave y con antecedentes de consumo de antipalúdicos previo al diagnóstico, 
en contrate con mujeres jóvenes con manifestaciones clínicas leves y en el perfil 5, pacientes de mayor edad, con corto tiempo de evolución de la enfermedad y presencia de sintomatología florida, propia de un cuadro agudo de paludismo. No se dispuso de información sobre el tiempo de permanencia en zona endémica de los pacientes en estos dos grupos, lo cual hubiera sido útil para definir el posible papel de la inmunidad adquirida como factor que influye en la gravedad de la presentación clínica del paludismo. El desarrollo de cierto grado de inmunidad en población adulta residente en zonas endémicas, después de múltiples exposiciones a la infección, puede disminuir la frecuencia de los episodios palúdicos, reducir la gravedad de los síntomas y signos, o ambos (29). Algunos estudios proponen que las diferencias en la presentación clínica pueden reflejar heterogeneidad en la virulencia del parásito (30), pero es poco probable que esto pueda explicar las diferencias observadas debido a que las poblaciones de parásitos circulantes en los dos municipios estudiados son bastante homogéneas (31).

Si bien el objetivo del presente estudio no fue identificar variables pronósticas de la falla del tratamiento, algunas de las descritas han sido identificadas en otros estudios como posibles factores predictores clínicos de falla terapéutica o que permiten clasificar y estratificar a pacientes candidatos para el uso de determinado antipalúdico. Así, por ejemplo, se han identificado factores predictores clínicos de falla terapéutica como edad menor de cinco años, fiebre, tamaño normal del bazo, desnutrición, niveles de parasitemia elevados, presencia de gametocitos, nivel de hemoglobina bajo, antecedente de episodios de paludismo previos recientes y el uso reciente de cloroquina. Con la descripción de estas características se pudo concluir que en algunos países de África, donde es común el uso de cloroquina, como en Uganda, se debe recomendar el uso de este medicamento en pacientes mayores pero es necesario utilizar otros agentes antipalúdicos en niños $(30,32,33)$.

Las debilidades del estudio incluyen el sesgo de memoria presente en el momento de recolectar la información y la baja frecuencia de pacientes con respuesta clínica adecuada, lo cual no permitió hacer comparaciones con el grupo de pacientes con falla del tratamiento.

Aunque el seguimiento por 14 días permite identificar altos niveles de fallas al tratamiento, como en efecto ocurrió en este estudio, no se puede descartar que algunos de los pacientes que presentaron respuesta clínica adecuada al día 14 hubiesen presentado falla terapéutica si se hubiera realizado seguimiento hasta el día 28 . Esto constituye una limitación adicional de este estudio.

En conclusión, el análisis multivariado permitió agrupar a los pacientes en cinco perfiles diferentes, de acuerdo con características clínicas y de laboratorio, los cuales corresponden a las descripciones encontradas en la literatura sobre la presentación habitual del paludismo no complicado.

En la revisión de la literatura científica indexada no fue posible encontrar estudios publicados que utilicen, como en este caso, el análisis multivariado por correspondencias múltiples que incorpora direccionalidad y que incluyen a la respuesta terapéutica como variable evaluada. Esta aproximación analítica permite agrupar a los pacientes con determinadas características clínicas cuyas diferencias podrían explicarse en relación con sus antecedentes personales, características eco-epidemiológicas o con diferencias en la patogénesis de la malaria y en las respuestas del huésped humano ante la infección, lo cual no es posible con análisis más simples.

Este tipo de estudios permite describir una línea de base a partir de la cual se pueden diseñar programas de vigilancia para identificar factores clínicos, epidemiológicos y de laboratorio relacionados con fallas a los tratamientos convencionales o identificar cambios en las dinámicas en la presentación de la enfermedad en una zona específica. La información obtenida a partir de ellos sirve como punto de partida al momento de describir la presentación clínica de el paludismo no complicado en el marco de la respuesta al tratamiento con los diferentes antipalúdicos utilizados, aporta información de utilidad para los estudios in vivo de eficacia de los medicamentos 
antipalúdicos y permite generar nuevas hipótesis de investigación. Aunque la población de estudio corresponde a un área geográfica muy particular y específica, guarda semejanzas con la población presente en otras regiones endémicas del país, lo cual permitiría replicar la metodología utilizada y evaluar su validez externa.

Finalmente, los resultados confirman el alto grado y amplia diseminación de la resistencia de $P$. falciparum a la cloroquina en Colombia y específicamente en una de las áreas de mayor transmisión de malaria en el país, como lo es el departamento de Córdoba.

\section{Agradecimientos}

Los autores agradecen a Liliana Jazmin Cortés y Sandra Valois del Grupo de Parasitología de la Red Nacional de Laboratorios del Instituto Nacional de Salud, quienes realizaron el control de calidad de las láminas para el diagnóstico parasitológico.

Los autores agradecen la colaboración recibida de las siguientes personas e instituciones para la realización del trabajo de campo: Carlos Usta, coordinador del Programa ETV de la Secretaría Departamental de Salud de Córdoba; Freddy Oviedo, Secretario Municipal de Salud de Tierralta; Uriel Negrete, César Gustavo Palomino, Edenia Martínez, Cielo Pastrana y Eliécer Hernández, microscopistas de Tierralta, Córdoba. A Jairo Pereira R., Secretario Municipal de Salud de Puerto Libertador; Severiano Velásquez Pimienta, Jairo Doria Hernández y Manuel López Hoyos, microscopistas de la empresa asociativa de trabajo DIFUSANJOR.

\section{Conflictos de interés}

Los autores declaran que durante las diferentes fases de realización de este estudio, no incurrieron en conflictos de interés de ninguna naturaleza que hubiesen podido afectar sus resultados.

\section{Financiación}

Este estudio fue financiado con recursos del Fondo de Investigaciones en Salud, Ministerio de la Protección Social-Colciencias, proyecto código 2104-04-11813, contrato No. RC 134-2002 y del Instituto Nacional de Salud.

\section{Referencias}

1. Warrell DA. Clinical features of malaria. En: Warrell DA, Gilles HM, editores. Essential malariology. 4 ed. London: Arnold; 2002. p.191-205.

2. Mombo LE, Ntoumi F, Bisseye C, Ossari S, Lu CY, Nagel RL, et al. Human genetic polymorphisms and asymptomatic Plasmodium falciparum malaria in Gabonese schoolchildren. Am J Trop Med Hyg. 2003;68:186-90.

3. World Health Organization. Management of severe malaria. A practical handbook. Second edition. Geneva: WHO; 2000.

4. Artavanis-Tsakonas K, Tongren JE, Riley EM. The war between the malaria parasite and the immune system: immunity, immunoregulation and immunopathology. Clin Exp Immunol. 2003;133:145-52.

5. Greenwood BM. Asymptomatic malaria infections do they matter? Parasitol Today. 1987;3:206-14.

6. Wongsrichanalai C, Pickard AL, Wernsdorfer WH, Meshnick SR. Epidemiology of drug-resistant malaria. Lancet Infect Dis. 2002;2:209-18.

7. Moore DV, Lanier JE. Observations on two Plasmodium falciparum infections with an abnormal response to chloroquine. Am J Trop Med Hyg. 1961;10:5-9.

8. Espinal CA, Cortés GT, Guerra P, Arias AE. Sensitivity of Plasmodium falciparum to antimalarial drugs in Colombia. Am J Trop Med Hyg. 1985;34:675-80.

9. Organización Panamericana de la Salud. Evaluación de la eficacia terapéutica de los medicamentos para el tratamiento de paludismo por Plasmodium falciparum sin complicaciones en las Américas. PAHO/HCP/HCT/ 113/98. Washington DC: Organización Panamericana de la Salud; 1998.

10. Osorio LE, Giraldo LE, Grajales LF, Arriaga AL, Andrade AL, Ruebush TK $2^{\text {nd }}$, et al. Assessment of therapeutic response of Plasmodium falciparum to chloroquine and sulfadoxine-pyrimethamine in an area of low malaria transmission in Colombia. Am J Trop Med Hyg. 1999;61:968-72.

11. López YL, Arroyave A, Salazar A. Evaluación de la resistencia in vivo a medicamentos antimaláricos. El Bagre, Antioquia, 1998. Revista Epidemiológica de Antioquia. 1999;24:181-93.

12. Blair S, Lacharme L, Carmona J, Tobón A. Resistencia del Plasmodium falciparum a los antimaláricos en Urabá y Bajo Cauca Antioqueño, 1998. Revista Epidemiológica de Antioquia. 1999; 24:207-15.

13. Castillo CM, Osorio LE, Palma GI. Assessment of therapeutic response of Plasmodium vivax and Plasmodium falciparum to chloroquine in a malaria transmission free area in Colombia. Mem Inst Oswaldo Cruz. 2002;97:559-62. 
14. Ministerio de Salud de Colombia. Guía de atención para el diagnostico y tratamiento de la malaria. Resolución No. 0412 de 2000, sección 37. [Consultado: 22 de diciembre de 2006]. Disponible en: http:// www.medicosgeneralescolombianos.com/Malaria.htm

15. Echeverri M, Tobón A, Álvarez G, Carmona J, Blair S. Clinical and laboratory findings of Plasmodium vivax malaria in Colombia, 2001. Rev Inst Med Trop Sao Paulo. 2003;45:29-34

16. González L, Guzmán M, Carmona-Fonseca J, Lopera T, Blair S. Características clínico epidemiológicas de 291 pacientes hospitalizados por malaria en Medellín (Colombia). Acta Médica Colombiana. 2000;25:163-70.

17. Instituto Nacional de Salud. Subdirección de Epidemiología y Laboratorio Nacional de Referencia División Centros de Control de Enfermedades. SIVIGILA. Comportamiento por regiones de la malaria en el 2001. Semana Epidemiológica No. 01. Bogotá, D.C.: Instituto Nacional de Salud; 2002.

18. Instituto Nacional de Salud. Subdirección de Epidemiología y Laboratorio Nacional de Referencia División Centros de Control de Enfermedades. SIVIGILA. Situación de la malaria en Colombia, semana epidemiologica 44 del año 2003. Semana Epidemiológica No. 44. Bogotá, D.C.: Instituto Nacional de Salud; 2003.

19. Mendoza NM, Nicholls RS, Olano VA, Cortés LJ, Raad J. Manejo integral de la malaria. 1 ed. Bogotá D.C.: Instituto Nacional de Salud; 2000.

20. Escoffier B, Pages J. Análisis factoriales simples y múltiples. Objetivos, métodos, interpretación. Bilbao: Servicio editorial de la Universidad del País Vasco; 1990. p.49-69.

21. Lebart L, Morineau A, Piron M. Statistique exploratoire multidimensionelle. Paris: Dunod; 1995. p.108-42.

22. Trape JF, Rogier C. Combating malaria morbidity and mortality by reducing transmission. Parasitol Today. 1996;12:236-40

23. Boisier P, Jambou R, Raharimalala L, Roux J. Relationship between parasite density and fever risk in a community exposed to a low level of malaria transmission in Madagascar highlands. Am J Trop Med Hyg 2002;67:137-40.
24. Osorio L, Todd J, Bradley D. Ausencia de malaria asintomática en escolares de Quibdo, Chocó. Biomédica. 2004;24:13-9.

25. González IJ, Padilla JO, Giraldo LE, Saravia NG. Eficacia de la amodiaquina y la sulfadoxina/pirimetamina en el tratamiento de la malaria no complicada por Plasmodium falciparum en Nariño, Colombia, 1999-2002. Biomédica. 2003;23:38-46.

26. Warrell DA, Turner GD, Francis N. Pathology and pathophysiology of human malaria. En: Warrell DA, Gilles HM, editores. Essential malariology. 4 ed. London: Arnold; 2002. p.236-51.

27. World Health Organization. WHO expert committee on malaria. WHO Technical Report Series 892. Twentieth Report. Geneva: WHO; 2000.

28. World Health Organization. Severe falciparum malaria. Trans R Soc Trop Med Hyg 2000; 94 (Suppl.1):S1-90.

29. Rogier C, Ly AB, Tall A, Cisse B, Trape JF. Plasmodium falciparum clinical malaria in Dielmo, a holoendemic area in Senegal: no influence of acquired immunity on initial symptomatology and severity of malaria attacks. Am J Trop Med Hyg. 1999;60:410-20.

30. Cox MJ, Kum DE, Tavul L, Narara A, Raiko A, Baisor $\mathbf{M}$, et al. Dynamics of malaria parasitaemia associated with febrile illness in children from a rural area of Madang, Papua New Guinea. Trans R Soc Trop Med Hyg. 1994;88:191-7.

31. Guerra AP, Knudson A, Nicholls S, Galindo JA, Ravid Z, Rahirant S, et al. Genotipificación de los genes msp1 (bloque2) y dhfr (codón 108) de Plasmodium falciparum en muestras de campo recolectadas en cuatro localidades endémicas de Colombia. Biomédica. 2006;26:101-12.

32. Hamer DH, MacLeod WB, Addo-Yobo E, Duggan CP, Estrella B, Fawzi WW, et al. Age, temperature, and parasitaemia predict chloroquine treatment failure and anaemia in children with uncomplicated Plasmodium falciparum malaria. Trans $\mathrm{R}$ Soc Trop Med Hyg. 2003;97:422-8.

33. Dorsey G, Kamya MR, Ndeezi G, Babirye JN, Phares CR, Olson JE, et al. Predictors of chloroquine treatment failure in children and adults with falciparum malaria in Kampala, Uganda. Am J Trop Med Hyg. 2000;62:686-92. 